Subsequent experiments will allow us to assess whether HSC administration can confer vasculoprotection.

\section{THE ROLES AND MECHANISMS OF PORE-FORMING TOXINS IN CARDIAC INJURY}

${ }^{1}$ Guozheng Wang*, ${ }^{2}$ Yasir Alhamdi, ${ }^{2}$ Cheng-Hock Toh. ${ }^{1}$ Sepsis Research Group, Institute of Infection and Global Health, University of Liverpool, Liverpool, L69 7ZB; ' ${ }^{2}$ niversity of Liverpool

\subsection{6/heartjnl-2017-311726.142}

Cardiac muscles and conduction system are very sensitive to interruption of plasma membrane integrity due to uncontrollable ion diffusion. There are many types of toxins that could bind to cell membrane and form pores to allow ion diffusion cross the membrane. However, the effects and underlying mechanisms of those pore-forming toxins on cardiac functions have not been systemically investigated.

In this work, we investigated the effects of extracellular histones (endogenous) and pneumolysin (exogenous) on cardiomyocytes and underlying molecular mechanisms using cultured cardiomyocytes and mouse models. We found that both toxins could bind to cardiomyocyte membrane and caused large inward currents which altered membrane potentials. This led arrhythmia of cultured cardiomyocytes and mice infused with isolated toxins. In addition, circulating troponins, a marker of cardiac injury, were significantly elevated and the contractile performance of cardiomyocytes was also reduced.

Further investigation, we found that the uncontrollable calcium influx mainly increased diastolic intracellular calcium concentration and resulted in calcium transient reduction. However, the calcium-dependent protein kinase C, particularly $P K C \pm$, was significantly activated by calcium influx. Consequently, the phosphorylation of Troponin I was enhanced, which is known to correlate to contractile reduction. In addition, the phosphorylation levels of P38 and JNK kinases, and endoplasmic reticulum (ER) stress levels were also increased. Those changes may contribute to the suppression of cardiomyocyte contractility too.

In conclusion, the endogenous pore-forming toxins, such as circulating histones released after extensive tissue injury, and exogenous pore-forming toxins released during bacterial infection, may play important roles in the development of cardiac complications in many types of critical illnesses. In certain scenario, such as sepsis, both endogenous and exogenous toxins may synergistically harm the heart and cause severe complications to lead to early death of patients. Therefore more intensive investigation should be carried out to clarify their effects in patients as well as their molecular mechanisms in order to develop effective tools to diagnose, prevent and treat those cardiac complications induced by pore-forming toxins.

\section{SELECTIVE INHIBITION OF PLASMA MEMBRANE CALCIUM ATPASE 4 IMPROVES VEGF-MEDIATED ANGIOGENESIS}

${ }^{1}$ Sathishkumar Kurusamy*, ${ }^{2,3}$ Dolores López-Maderuelo, ${ }^{4}$ Robert Little, ${ }^{1}$ David Cadagan, ${ }^{5}$ Aaron M Savage, 'Jude C Ihugba, ${ }^{1}$ Rhiannon R Baggott, ${ }^{6}$ Farjana B Rowther, ${ }^{2}$ Sara Martinez-Martinez, ${ }^{2,3,7}$ Pablo Gomez del Arco, ${ }^{1}$ Clare Murcott, ${ }^{8}$ Weiguang Wang, ${ }^{4}$ Delvac Oceandy, ${ }^{4,9}$ Ludwig Neyses, ${ }^{5}$ Robert N Wilkinson, ${ }^{4}$ Elizabeth J Cartwright, 2,3 Juan Miguel Redondo, 'Angel Luis Armesilla. 'Cardiovascular Molecular Pharmacology Laboratory, School of Pharmacy; ${ }^{2}$ Gene Regulation in Cardiovascular Remodelling and Inflammation Group, Centro Nacional de Investigaciones Cardiovasculares, Madrid, Spain; ${ }^{3}$ CIBERCV; ${ }^{4}$ Division of Cardiovascular Sciences, University of Manchester, Manchester Academic Health Sciences Centre, Manchester, UK; ${ }^{5}$ Department of Infection, Immunity and Cardiovascular Disease and Bateson Centre, University of Sheffield, UK; ${ }^{6}$ Brain Tumour UK Neuro-oncology Research Centre; ${ }^{7}$ Department of Molecular Biology, Universidad Autonoma de Madrid, Madrid, Spain; ${ }^{8}$ Oncology Laboratory, Research Institute in Healthcare Science, Faculty of Science and Engineering, University of Wolverhampton, Wolverhampton, UK; ${ }^{9}$ University of Luxembourg

\subsection{6/heartjnl-2017-311726.143}

Ischaemic cardiovascular disease is the leading cause of death worldwide. Therapeutic angiogenesis aims to stimulate the growth of new blood vessels from pre-existing ones to reperfuse ischaemic tissues. Our laboratory is characterising the molecular mechanisms that regulate activation of the calcineurin/NFAT pathway during VEGF-induced angiogenesis. We recently showed that the Plasma Membrane Calcium ATPase 4 (PMCA4) negatively regulates angiogenesis by establishing a molecular interaction with calcineurin. The identification of aurintricarboxylic acid (ATA) as an inhibitor of PMCA4 prompted us to hypothesise that ATA will enhance VEGFinduced angiogenesis.

Consistent with this hypothesis, we demonstrate in this work that inhibition of PMCA4 by treatment with ATA significantly increases the activity of calcineurin/NFAT signalling, and the subsequent expression of the NFAT-dependent, proangiogenic protein RCAN1.4 in VEGF-stimulated endothelial cells. Targeting PMCA4 with ATA significantly reduces the level of membrane-associated calcineurin, and the amount of calcineurin co-precipitated with PMCA4 in immunoprecipitation assays, indicating that ATA promotes disruption of the PMCA4/calcineurin interaction. ATA robustly enhances endothelial cell motility, and in vitro and in vivo blood vessel formation, with no harmful effects to the cells. Interestingly, incubation of HUVECs with low concentration of ATA had no effect on the viability of the cells or the development of zebra fish embryos. However, higher ATA concentrations were associated with cellular and embryo toxicity.

Our study provides evidence for the therapeutic potential of targeting endothelial PMCA4 to improve VEGF-based proangiogenic interventions, and highlights possible clinical applications for PMCA4 inhibitors in the treatment of ischaemic cardiovascular disease. 